\title{
Fatores associados ao uso de diretrizes clínicas em operadoras de planos de saúde e prestadores de serviços hospitalares no campo da Saúde Suplementar no Brasil
}

\author{
Factors associated with the use of clinical guidelines \\ by health plan operators and their inpatient care providers
}

M argareth Crisóstomo Portela ${ }^{1}$

Sheyla M aria Lemos Lima ${ }^{1}$

M aurício Teixeira Leite de Vasconcellos ${ }^{4}$

Claudia Caminha Escosteguy ${ }^{3}$

Vanja M aria Bessa Ferreira ${ }^{2}$

M iguel Murat Vasconcellos ${ }^{1}$

Cláudia Brito ${ }^{1}$

${ }^{1}$ Departamento de

Administração e

Planejamento em Saúde,

Escola Nacional de Saúde

Pública, Fundação Oswaldo

Cruz. Rua Leopoldo

Bulhões 1480/724,

M anguinhos. 21041-210

Rio deJaneiro RJ.

mportela@ensp.fiocruz.br

${ }^{2}$ Secretaria Estadual de

Saúdedo Rio deJaneiro.

${ }^{3} \mathrm{H}$ ospital dos Servidores

do Estado do Rio deJaneiro.

${ }^{4}$ Escola $\mathrm{N}$ acional de

Ciências Estatísticas,

Fundação Instituto

Brasileiro de Geografia e

Estatística.
Abstract This paper is aimed at identifying factors related to the use of clinical guidelines by health plan operators and their inpatient care providers in the supplementary care sector in Brazil from theviewpoint of managers. The study is based on two national surveys: one involving 90 health plan operators randomly selected from a universe of 1573 , oriented towards characterizing the implementation of clinical guidelines and other tools of clinical governance; and another involving 74 hospitals, selected from a universe of 3817 inpatient care providers, aimed at capturing micro-regulation mechanisms applied by health plan operators and their repercussions on hospital practices. U se of clinical guidelines was reported by $32.3 \%$ of the health plan operators, and $51.6 \%$ of the hospitals interviewed. Among the first, geographical location, type and size of the organization were independently associated with use of clinical guidelines while among the hospitals size, complexity and use of other tools of clinical governance were independently associated with the dependent variable. The results show how incipient and unsatisfactory the intervention of thehealth plan and hospital managements still is with regard to the quality of health services offered to the beneficiaries.

Key words Clinical guidelines, Clinical governance, $\mathrm{H}$ ealth plan operators, $\mathrm{H}$ ospitals
Resumo Este artigo objetiva identificar fatores associados ao uso de diretrizes clínicas, segundo percepção dedirigentes respondentes, em operadoras de planos de saúde e prestadores de serviços hospitalares no campo da saúde suplementar no Brasil. U tiliza dados de dois inquéritos de abrangência nacional: um envolvendo 90 operadoras selecionadasaleatoriamentedeum universo de 1.573 , voltado para a caracterização da implementação de di retrizes clínicas e outros instrumentos e práticas de gestão da clínica; e outro envolvendo 74 hospitais, selecionados de um universo de 3.817 prestadores deserviços hospitalaresa planos desaúde, destinado a apreender os mecanismos de microrregulação das operadoras e suas possíveis repercussões sobre as práticas dos hospitais. Referiram o uso de diretrizes clínicas 32,3\% das operadoras e $51,6 \%$ dos hospitais. Entre as primeiras, mostraram-se independentemente associados ao uso dediretrizes, localização geográfica, tipo e porte da operadora, enquanto que, entre os hospitais, porte, complexidade e uso de outras ferramentas da gestão da clínica. Os resultados evidenciam 0 quanto ainda éincipientee insatisfatória a gestão, por operadoras e hospitais, sobre a qualidade dos cuidados à saúde providos aos pacientes. Palavras-chave Diretrizes clínicas, Gestão da clínica, O peradoras de planos de saúde, Hospitais, Saúde suplementar 
Introdução

A área da assistência à saúde e, mais especificamente, da assistência médica, tem sido marcada, desde a década de 1990, por uma crescente preocupação com o estímulo à efetiva utilização de práticas endossadas pelo conhecimento científico corrente. A motivação maior é a perspectiva de melhoria da qualidade da assi stência mas, de forma progressiva, também tem tido importância a perspectiva de alocação mais eficiente de recursos comumente limitados.

A valorização da medicina baseada na evidência científica serve como cenário para o de senvolvimento de diretrizes clínicas. Ela corresponde à integração da evidência proporcionada por pesquisas clinicamenterelevantes, da experiência do clínico e das preferências do paciente ${ }^{1}$. As diretrizes clínicas, por sua vez, constituem-se em posicionamentos ou recomendações (statements) sistematicamente desenvolvidos para orientar médicos e pacientes acerca dos cuidados de saúde apropriados, em circunstâncias clínicas específicas ${ }^{2}$. Contemplam indicações e contraindicações, bem como benefícios esperados eriscos do uso de tecnologias em saúde (procedimentos, testes diagnósticos, medicamentos, etc.) para grupos de pacientes definidos.

A discussão em torno de diretrizes clínicas origina-se da constatação de variações dos padrões de prática e de utilização de serviços de saúde, de uso inapropriado de serviços e da incerteza acerca dos resultados obtidos pelo uso ou não uso de serviços ou procedimentos. $\mathrm{Na}$ medida em que as diretrizes baseiam-se no conhecimento científico, estimativas dos resultados esperadosejulgamento profissional corrente, elas claramente têm um papel na garantia e avaliação de qualidade dos cuidados de saúde.

Hoje, é internacionalmente aceita a pressuposição de quea implementação de diretrizes para a prevenção, diagnóstico, tratamento e reabilitação de doenças, definidas a partir da evidência científica disponível acerca da eficácia e efetividade de intervenções, produz melhores resultados na população assistida ${ }^{3,4}$. Além disso, o desenvolvimento de diretrizes para a assistência à saúde propicia uma utilização mais racional dos re cursos disponíveis, o que é fundamental para diversos sistemas de saúde, mais ou menos dependentes de recursos públicos, em contextos de maior ou menor restrição de recursos.

Apesar do reconhecimento de que a utilização de diretrizes clínicas contribui para a melhoria da atenção prestada, sua efetiva aplicação ain- da é insatisfatória, havendo resistência por parte dos profissionais de saúde, especialmente médicos, e pacientes. Tem sido uma preocupação presente na literatura internacional a identificação decaracterísticas facilitadoras do seu uso eestratégias efetivas para a sua disseminação.

Estudos focalizando a identificação de fatores facilitadores do uso de diretrizes clínicas indicam a sua aplicação mais bem-sucedida em áreas específicas da atenção médica, tais como a cardiológica, a oncológica e a oftalmológica. Também apontam a importância das diretrizes clínicas serem atuais, simples, precisas, não controversas e não demandantes de mudanças importantes na prática cotidiana ${ }^{5-7}$. Sugerem ainda que as diretrizes devem ser desenvolvidas e/ou endossadas por organizações profissionais e por médicos reconhecidos como líderes nas áreas em foco ${ }^{6}$. Além disso, indicam a maior efetividade de estratégias de disseminação ativas, calcadas em intervenções multifacetadas, incluindo auditoria e feedback, consensos locais ou propagan$\mathrm{da}$, eencontros educacionais interativos $\mathrm{s}^{8}$, ou ainda outras práticas de gestão da clínica, tais como a gestão do caso 9 .

Estudos focalizando características facilitadoras do uso de diretrizes clínicas, pertinentes às organizações de saúde, têm sido menos freqüentes. Ainda assim, achados, entre operadoras de planos de saúde, indicam o papel do tipo e tamanho daquelas organizações ${ }^{10,11}$, bem como, entre hospitais, al guma diferenciação associada ao porte ${ }^{12}$.

No âmbito da saúde suplementar no Brasil, em um momento em que a atuação da Agência Nacional de Saúde Suplementar (ANS) tem sido fortemente marcada pela preocupação com a regulação da assistência, com vistas à sua qualificação, vale considerar o seu papel potencial na indução direta ou indireta do uso de diretrizes clínicas, seja por operadoras de planos de saúde, seja pelos prestadores de serviços hospitalares e ambulatoriais.

Este artigo visa a identificar fatores associados ao uso de diretrizes clínicas, segundo percepção de dirigentes respondentes, em operadoras de planos de saúde e prestadores de serviços hospitalares no campo da saúde suplementar. Não se trata de um estudo sobre a real utilização de diretrizes pelas operadoras e prestadores hospital ares atuantes na saúde suplementar, mas fornece indicações acerca deseu uso, tendo em vista o pressuposto quequaisquer iniciativas organizacionais, para que se tornem realidade, precisam estar incorporadas como uma dimensão da gestão da organização e, como tal, no discurso de seus dirigentes. 


\section{Métodos}

As análises aqui apresentadas partem de dados obtidos em dois inquéritos de abrangência nacional: um voltado para a caracterização da implementação de diretrizes clínicas e outros instrumentos e práticas de gestão da clínica nas operadoras de planos de saúde ${ }^{13}$; e outro destinado a apreender, considerando a perspectiva de prestadores de serviços hospitalares na saúde suplementar, os mecanismos de microrregulação praticados pelas operadoras de planos de saúde e suas possíveis repercussões sobre as práticas dos hospitais ${ }^{14}$. Em ambos os estudos, foi considerada, exclusivamente, a informação prestada pelos entrevistados, não ten do havido nenhum tipo de validação dessa informação.

Os dois estudos, apesar de orientados a captar dados de unidades de observação diferentes, apresentam concepções bem semelhantes, com desenho amostral complexo.

0 primeiro foi realizado no período entre novembro de 2004 e julho de 2005, tendo como população-alvo o conjunto de 1.573 operadoras de planos de saúde que constava do cadastro de seleção fornecido pela ANS atualizado até 18 de agosto de 2004. A pesar da definição da estratificação em termos das macrorregiões e dos segmentos de mercado, a assimetria da distribuição do número de beneficiários foi um problema que requereu atenção, para evitar um tamanho de amostra muito grande ou instabilidade nas estimativas em amostras pequenas. Assim, aplicouse 0 método de Hedlin ${ }^{15}$ para obter a amostra mais precisa dentre as possíveis amostras de tamanho 80. Estratos naturais foram constituídos combinando macrorregiões e segmentos de mercado, sendo feita, nestes, uma estratificação estatística de porte que, em função das características da distribuição do número de beneficiários em cada estrato natural, gerou estratos certos e estratos amostrados. A pós a criação dos estratos certos nos estratos naturais, o tamanho de amostra restante de cada estrato natural foi alocado, no caso de existir, ao seu estrato amostrado, sendo assegurado um tamanho de amostra maior ou igual a 2 em cada estrato amostrado, o que aumentou o tamanho total da amostra para 112 operadoras. Nos estratos amostrados, foi feita uma seleção equiprovável das operadoras por meio do algoritmo de Hájek ${ }^{16}$, ou seja, foi selecionada uma amostra al eatória simples de operadoras. Durante o trabalho de campo, diversas ocorrências geraram a necessidade de substituição de unidades selecionadas que não puderam ser en- trevistadas. Para a substituição, unidades em estratos certos que não prestaram informações foram transferidas para estratos amostrados. No total, foram contatadas 170 operadoras, das quais 25 apresentavam erro de cadastro (operadora não-encontrada, comprada ou fusão com outra, falida, etc.), 55 recusaram-se a prestar informações e as noventa restantes foram entrevistadas, compondo assim a amostra final do estudo.

0 segundo estudo foi realizado no período entre setembro de dezembro de2006, tendo como universo o conjunto de 3.817 unidades hospitalares prestadoras de serviços às operadoras de planos de saúde, definido a partir do cruzamento do Cadastro dePrestadores deServiçosa Planos deSaúde, da Agência Nacional de Saúde Suplementar (ANS), com o Cadastro Nacional de Estabelecimentos de Saúde (CNES) em julho de 2006. Os estratos naturais da amostra foram constituídos combinando as macrorregiões geográficas e o tipo de hospital (geral ou especializado), sendo a alocação do tamanho total da amostra nos estratos naturais feita de forma proporcional ao seu número deleitos. Paraaumentar a eficiência da amostra em cada estrato natural, foi aplicado o algoritmo de Hedlin ${ }^{15}$ para determinar o ponto de corte (número de leitos) entre um estrato-certo (take-all) e um estrato amostrado (take-some), de forma a minimizar a variância do número de leitos, dado um tamanho préfixado de amostra no estrato natural. 0 tamanho da amostra, inicialmente fixado em 75 hospitais, acabou sendo de 83 unidades ( 28 em estratos certos e 55 em estratos amostrados), por causa dos arredondamentos efetuados em cada estrato. N os estratos amostrados, adotou-se um mecanismo de amostragem inversa ${ }^{17}$. Ao todo, foram contatados 95 hospitais: 28 deestratos certose 67 que constavam da lista inicial (55) ou de substituição de estratos amostrados (12). Entre os 28 hospitais que constavam de estratos certos, ocorreram três exclusões de unidades que não prestavam serviços no campo da saúde suplementar e quatro recusas (sem possibilidade de substituição), sendo entrevistados 21 hospitais. Por outro lado, entre os 67 prestadores de serviços hospitalares de estratos amostrados, foram registradas catorze recusas e entrevistadas 53 unidades, valendo sublinhar a impossibilidade de duas substituições em dois estratos amostrados com listas-reserva esgotadas. Desse modo, a amostra final pesquisada resultou em 74 hospitais.

Em ambos os estudos, foram elaborados questionários estruturados incluindo variáveis de caracterização das organizações focal izadas e re- 
lativas ao uso de diretrizes clínicas e outras práticas de gestão da clínica. As coletas de dados foram realizadas através de entrevistas, previamente agendadas, por pesquisadores de campo treinados, junto aos dirigentes das operadoras responsáveis pelo relacionamento com os prestadores hospital ares eaos dirigentes hospital ares responsáveis pela área médico-assistencial. Os dados foram digitados pelos pesquisadores de campo em formulário el etrônico earmazenadosem bancos de dados.

Para a identificação de fatores associados ao uso de diretrizes clínicas percebido pelos respondentes em cada um dos estudos, foram realizadas análises bivariadas com aplicação do teste qui-quadrado $\left(\chi^{2}\right)$ e modelos de regressão logística. Considerando variáveis coletadas nos estudos utilizados, explorações centraram-se no papel explicativo potencial de variáveis demográficas relativas às operadoras - macrorregião de localização da sede, segmento do mercado e porte(número de beneficiários) - ou aos hospitais macrorregião de localização, porte (número de leitos), complexidade, relações com operadoras de planos de saúde e atendimento ao SUS -, bem como da presença de outras práticas de gestão da clínica. No último caso, o racional sedimentase na importância da articulação de estratégias diversas de gestão da clínica para a obtenção de resultados assistenciais mais efetivos e na perspectiva de que as diretrizes clínicas são em si instrumentos da gestão da clínica, com o papel especial de embasar a aplicação de outros instrumentos/ferramentas, tais como a gestão do caso, a gestão de patologias, etc.

Especificamente em relação aos hospitais, merece ser destacada a variável explicativa“complexidade assistencial". Foram construídas cinco classes diferentes a partir da informação registrada no Cadastro Nacional de Estabelecimentos de Saúde/CNES do M inistério da Saúde sobreo tipo do hospital (geral versusespecializado) e da informação contida no questionário da pesquisa sobre os serviços existentes, equipamentos compatíveis e a realização de procedimentos de al ta complexidade na internação (PAC). Os hospitais especializados foram divididos em duas classes: especializado com UTI eespecializado sem UTI. A classe dos hospitais mais complexos, denominada de Geral II, incluiu hospitais gerais necessariamente com presença de UTI, além da realização de procedimento de alta complexidade na internação em ao menos uma das seguintes áreas: cardiovascular, cirurgia oncológica, neurocirurgia, ortopedia, transplantes e neona- tologia, com presença de equipamentos em uso compatíveis. Os demais hospitais gerais de mé dia e baixa complexidade e hospitais de clínicas básicas foram reunidos em duas classes segundo a presença ou não de unidade de tratamento intensivo, quais sejam as classes, Geral I com UTI e Geral I sem UTI.

Sendo o desenho das amostras complexo, a estimação de variâncias e da significância de parâmetros dos modelos estatísticos não pode ser feita por métodos clássicos, que pressupõem que as observações da amostra são independentes e igualmente distribuídas. É fato que a estimação pontual de porcentagens, totais, médias e parâmetros de modelos, feita com emprego dos fatores de expansão, não apresenta qualquer tipo de viés ou erro, mas variâncias, desvios-padrão, erros-padrão e, portanto, os testes de hipóteses devem considerar, além dos fatores de expansão, a estratificação usada, visto que os estratos certos não contribuem para a variância.

Nesse sentido, as análises aqui apresentadas foram realizadas com o pacote estatístico SUDAAM, na versão 9 com interface SAS-callable, capaz de dar conta de dados oriundos de amostras complexas.

\section{Resultados}

No primeiro estudo, a amostra resultante de noventa operadoras, expandida, corresponde a 1.572 das 1.573 operadoras da população contemplada - uma das seguradoras recusou-se a prestar informações e não pôde ser substituída porque era única no estrato. No conjunto de tais operadoras, ressaltam-se a predominância da medicina de grupo eo peso relevante do número de beneficiários do segmento das seguradoras, embora represente o menor percentual no conjunto de operadoras. Destaca-se, ainda, a grande concentração de operadoras na região Sudeste $(59,5 \%)$, em contraposição às demais regiões Sul $(17,0 \%)$, Nordeste $(13,0 \%)$, Centro-O este $(7,2 \%)$ e N orte $(3,3 \%)$.

No outro estudo, a amostra final de prestadores hospitalares na saúde suplementar, constituída por 74 unidades, corresponde a um universo estimado de 3.799 hospitais, em contraponto ao universo programado de 3.817 hospitais. A maior concentração de hospitais foi registrada na região Sudeste, com $41,5 \%$ dos hospitais, seguida pela região Sul $(21,6 \%)$, Nordeste $(19,0 \%)$, Centro-Oeste $(12,9 \%)$ e N orte $(5,0 \%)$.

Em termos gerais, 32,3\% das operadoras de 
planos de saúde e $51,6 \%$ dos hospitais prestadores de serviços na saúde suplementar declararam o uso de diretrizes clínicas.

Fatores associados ao uso de diretrizes clínicas nas operadoras de planos de saúde

Considerando os dados concernentes às ope radoras de planos de saúde e o nível de signifi- cância estatística $\alpha=0,05$, as análises bivariadas indicaram a associação da declaração de uso de diretrizes clínicas com as variáveis macrorregião do país, segmento do mercado de saúde suplementar e porte da operadora (Tabela 1).

0 uso de diretrizes clínicas foi reportado por $45,5 \%$ das operadoras com sedeno Sudeste, $37,5 \%$ das operadoras com sede no Centro- 0 este e $22,5 \%$ das operadoras com sede no Sul do país.

Tabela 1. Distribuição do uso de diretrizes clínicas reportado por operadoras de planos de saúde na saúde suplementar $(n=1572)$ segundo variáveis selecionadas. Análise bivariada. Brasil, 2005.

\begin{tabular}{|c|c|c|c|c|c|c|c|}
\hline \multirow[b]{3}{*}{ Variável } & \multirow[b]{3}{*}{$\mathrm{n}$} & \multirow[b]{3}{*}{$\%$} & \multicolumn{4}{|c|}{ Uso de diretrizes } & \multirow{3}{*}{$\begin{array}{l}\text { Teste- } \\
\chi^{2}(p)\end{array}$} \\
\hline & & & \multicolumn{2}{|c|}{$\underset{(n=507)}{\operatorname{Sim}}$} & \multicolumn{2}{|c|}{$\begin{array}{c}\text { N ão } \\
(n=1065)\end{array}$} & \\
\hline & & & $n$ & $\%$ & $\mathrm{n}$ & $\%$ & \\
\hline M acrorregião & & & & & & & 0,0173 \\
\hline Norte & 59 & 3,7 & 0 & 0,0 & 59 & 100,0 & \\
\hline Nordeste & 248 & 15,8 & 1 & 0,4 & 247 & 99,6 & \\
\hline Sudeste & 892 & 56,7 & 406 & 45,5 & 486 & 54,5 & \\
\hline Sul & 267 & 17,0 & 60 & 22,5 & 207 & 77,5 & \\
\hline Centro-Oeste & 107 & 6,8 & 30 & 37,5 & 67 & 62,5 & \\
\hline Segmento do mercado de saúde suplementar & & & & & & & 0,0246 \\
\hline Autogestão & 326 & 20,7 & 125 & 38,2 & 201 & 61,8 & \\
\hline Cooperativa médica & 370 & 23,5 & 95 & 25,8 & 275 & 74,2 & \\
\hline Filantrópico & 124 & 7,9 & 8 & 6,5 & 116 & 93,5 & \\
\hline M edicina de grupo & 739 & 47,0 & 278 & 37,7 & 461 & 62,3 & \\
\hline Seguradora & 13 & 0,8 & 1 & 7,7 & 12 & 92,3 & \\
\hline Porte da operadora & & & & & & & 0,0184 \\
\hline > 20.000 beneficiários & 550 & 35,0 & 303 & 55,0 & 248 & 45,0 & \\
\hline$\leq 20.000$ beneficiários & 1022 & 65,0 & 205 & 20,0 & 817 & 80,0 & \\
\hline Gestão de patologia & & & & & & & 0,3346 \\
\hline Sim & 523 & 33,3 & 225 & 43,1 & 298 & 56,9 & \\
\hline Não & 1049 & 66,7 & 282 & 26,9 & 767 & 73,1 & \\
\hline Gestão de caso & & & & & & & 0,2962 \\
\hline Sim & 455 & 28,9 & 98 & 21,5 & 357 & 78,5 & \\
\hline Não & 1117 & 71,1 & 409 & 36,7 & 708 & 63,3 & \\
\hline Estudos estatísticos da variação na prática médica & & & & & & & 0,2789 \\
\hline Sim & 478 & 30,4 & 216 & 45,1 & 262 & 54,9 & \\
\hline Não & 1094 & 69,6 & 291 & 26,7 & 803 & 73,3 & \\
\hline Sistema(s) de informação na área assistencial* & & & & & & & 0,5902 \\
\hline Sim & 1357 & 86,7 & 455 & 33,6 & 902 & 66,4 & \\
\hline Não & 209 & 13,3 & 46 & 22,0 & 163 & 78,0 & \\
\hline Pesquisa satisfação dos beneficiários & & & & & & & 0,3455 \\
\hline Sim & 660 & 42,0 & 268 & 40,7 & 392 & 59,3 & \\
\hline Não & 912 & 58,0 & 239 & 26,2 & 673 & 73,8 & \\
\hline Ouvidoria* & & & & & & & 0,2084 \\
\hline Sim & 934 & 59,7 & 369 & 39,6 & 564 & 60,4 & \\
\hline Não & 631 & 40,3 & 138 & 21,8 & 494 & 78,2 & \\
\hline Campanhas de promoção, prevenção e educação & & & & & & & 0,1856 \\
\hline $\operatorname{Sim}$ & 1108 & 70,5 & 425 & 38,3 & 683 & 61,7 & \\
\hline Não & 464 & 29,5 & 82 & 17,8 & 382 & 82,2 & \\
\hline
\end{tabular}

*Para essas variáveis, não se obteve resposta de todas as operadoras estudadas. 
$\mathrm{Na}$ região N ordeste, nenhuma das operadoras entrevistadas reportou o uso de diretrizes clínicas e, na região N orte, somente uma ( $0,4 \%)$ o fez.

Entre os segmentos do mercado de saúde suplementar, o uso de diretrizes clínicas foi declarado por $38,2 \%$ das empresas de autogestão, 37,7\% das empresas de medicina de grupo, $25,8 \%$ das cooperativasmédicas, 7,7\% das seguradoras e $6,5 \%$ das operadoras ligadas a entidades filantrópicas.

Utilizando-se como ponto de corte para o porte das operadoras o valor de 20 mil beneficiários, observou-se maior referência ao uso de diretrizes entre as operadoras do grupo de maior porte. No grupo de operadoras com mais de 20 mil beneficiários, $55 \%$ reportaram o uso, enquanto que somente $20 \%$ das operadoras do grupo com 20 mil beneficiários ou menos o fizeram.

As análises bivariadas não indicaram associações estatisticamente significativas entre declaração de uso de diretrizes clínicas e uso de gestão de patologia, de gestão do caso, de estudos estatísticos da variação na prática médica, de sistemas de informação na área assistencial e de pesquisas de satisfação dos beneficiários. Também não mostraram associações com a existência de Ouvidoria, nem com a realização de campanhas de promoção, prevenção e educação.

0 modelo de regressão logística (Tabela 2), por sua vez, apontou ef eitos independentes estatisticamente significantes sobre a probabilidade de uso de diretrizes clínicas, da localização geográfica da sede da operadora, do segmento do mercado, do porte da operadora e do uso de gestão de caso.
As chances de uso de diretrizes clínicas (versus não uso) entre operadoras das regiões $N$ orte e Nordeste mostraram-se nulas $(O R=0,00)$, comparadas com as observadas nas regiões Sudeste, Sul e Centro-Oeste, mantendo-se as demais variáveis constantes. A amostra não permitiu a diferenciação das chances de uso de diretrizes clínicas entre estas três regiões.

No que concerne aos segmentos do mercado de saúde suplementar, as empresas de autogestão, de medicina de grupo e filantrópicas terminaram sendo agrupadas como categorias de referência. As proporções de uso de diretrizes clínicas declarado são realmentesemel hantesnos dois primeiros casos, podendo a incapacidade de diferenciação das operadoras do segmento filantrópico dever-se ao tamanho da amostra. As chances de uso de diretrizes clínicas (versus não uso) nas cooperativas médicas e nas seguradoras mostraram-se, respectivamente, $81 \%$ e $96 \%$ menores do que nas operadoras do grupo de referência, controlando-se as demais variáveis.

Também expressiva na diferenciação das chances de uso de diretrizes clínicas nas operadoras de planos de saúde mostrou-se a variável porte, expressa em termos da dicotomização nas categorias " $\leq 20.000$ beneficiários" (referência) e ">20.000 beneficiários" ( $O R=12,89)$.

De forma surpreendente, as chances de declaração do uso de diretrizes clínicas foram 92\% menores nas operadoras que responderam realizar gestão de caso do que naquelas que referiram não realizar.

Tabela 2. M odelo de regressão logística explicativo do uso de diretrizes clínicos reportado por operadoras de planos de saúde ( $n=1572)$. Brasil, 2005.

\begin{tabular}{|c|c|c|c|c|c|}
\hline Variável & $\beta$ & Erro-padrão & $\mathrm{p}$ & Odds ratio & IC95\% \\
\hline Intercepto & $-0,93$ & 0,68 & 0,1789 & & \\
\hline \multicolumn{6}{|l|}{ M acrorregião (ref: Sudeste, Sul e Centro-O este) } \\
\hline Norte & $-10,27$ & 0,71 & 0,0000 & 0,00 & 0,$00 ; 0,00$ \\
\hline Nordeste & $-5,30$ & 1,07 & 0,0000 & 0,00 & 0,$00 ; 0,04$ \\
\hline \multicolumn{6}{|l|}{ Segmento do mercado (ref: outros*) } \\
\hline Cooperativa médica & $-1,67$ & 0,97 & 0,0904 & 0,19 & 0,$03 ; 1,31$ \\
\hline Seguradora & $-3,31$ & 1,47 & 0,0280 & 0,04 & 0,$00 ; 0,69$ \\
\hline $\begin{array}{l}\text { Operadora > } 20.000 \text { benef. (ref: até } 20.000 \text { benef.) } \\
\text { Gestão de patologia (ref: não) }\end{array}$ & 2,56 & 0,85 & 0,0041 & 12,89 & 2,$33 ; 71,28$ \\
\hline Sim & 1,89 & 1,14 & 0,1029 & 6,59 & 0,$68 ; 64,31$ \\
\hline \multicolumn{6}{|l|}{ Gestão de caso (ref: não) } \\
\hline Sim & $-2,57$ & 1,08 & 0,0212 & 0,08 & 0,$01 ; 0,67$ \\
\hline
\end{tabular}

*Autogestão, medicina degrupo efilantrópico. 
Finalmente, vale sublinhar a associação positiva e de el evada magnitude $(O R=6,59)$, mas não estatisticamente significante $(p=0,1029)$, da de claração do uso de diretrizes clínicas com a declaração da realização de gestão de patologia.

Fatores associados ao uso de diretrizes clínicas nos prestadores de serviços hospital ares da saúde suplementar

A Tabela 3 apresenta os resultados das análises bivariadas entre declaração do uso de diretrizes clínicas e potenciais variáveis explicativas no âmbito dos hospitais considerados. Baseado no teste $\chi^{2}$, foram identificadas asso ciações da variável dependentedeinteressecom macrorregião do país, a condição de hospital próprio de operadora de plano de saúde, o número de operadoras para as quais o hospital presta serviços, a complexidade assistencial, acreditação, presença de serviço/núcleo de qualidade, declaração da realização de gestão de patologia e de gestão do caso, existência de 0 uvidoria e realização de procedimentos de al ta complexidade (PAC) nas áreas de cirurgia oncológica, neurocirurgia, ortopedia, transplantes e neonatologia.

0 uso de diretrizes clínicas foi declarado por $62,7 \%$ dos hospitais do Sudeste, $50,2 \%$ dos hospitais do Sul, 39,4\% dos hospitais do N ordestee $19,9 \%$ dos hospitais do Centro-O este. Estranho pareceu o fato de $94,4 \%$ dos hospitais da região N orteterem respondido utilizar diretrizes, o que, entretanto, pode ter decorrido de inadequação da amostra.

Entre os hospitais próprios de operadoras, $99,3 \%$ declararam utilizar diretrizes, em contraposição a $48,1 \%$ nos hospitais não próprios, merecendo também destaque a associação positiva entre o uso de diretrizes e o número de operadoras para as quais o hospital presta serviços. No segmento de hospitais que presta serviços a mais de 50 operadoras, $99,4 \%$ deles referiram usar diretrizes clínicas.

Não foi possível rejeitar a hipótese de independência entrea declaração do uso de diretrizes eo porte do hospital $(p=0,1298)$. A tendência de maior uso entrehospitais com mais leitos é que brada pelos hospitais como 151 a 350 leitos.

Vale também ressaltar a clara associação do uso de diretrizes com a complexidade assistencial do hospital. A totalidade, $82,6 \%$ e $78,6 \%$ dos hospitais especializados com UTI, gerais II e gerais I com UTI, declarou utilizar diretrizes clínicas, respectivamente. Entreos hospitais gerais। e especializados sem UTI, tais índices caem para $29,9 \%$ e $20,8 \%$. Por outro lado, declararam o uso de diretrizes $99,4 \%$ dos hospitais com PAC de transplantes, 92,7\% com PAC de cirurgia oncológica, 89,2\% com PAC de neonatologia e 86,6\% com PAC de neurocirurgia. Surpreendentemente, PAC na área cardiovascular mostrou-se somente com uma associação borderline $(p=0,0742)$ com o uso de diretrizes, tendo $77,0 \%$ dos hospitais com tal PAC respondido usar diretrizes, contra 45,4\% dos hospitais sem PAC referirem o uso.

Deforma geral, os resultados da Tabela 3 não indicam muita relação do uso de diretrizes com o de outras estruturas ou prática de qualificação da assistência, sendo exceção importanteo elevado uso entre os hospitais que declararam realizar gestão de patologia $(80,4 \%)$ e gestão de caso $(90,4 \%)$.

0 modelo de regressão logística apresentado na Tabela 4 aponta que o porte intermediário (101-150 leitos), a presença dePAC detransplante e a declaração de realização de gestão de caso mostraram-se independentemente associados à declaração do uso de diretrizes no nível de significância $\alpha=0,05$.

Deve ser sublinhado que a opção de considerar as diversas classes de complexidade assistencial no modelo foi descartada em função da incapacidade da amostra de prover estimativas estáveis para elas e da superposição do registro de PAC com a classificação como hospital geral II. Preferiu-se, então, considerar simplesmente a presença de UTI (versus ausência) e explorar o efeito de diferentes PAC.

As chances de declaração do uso de diretrizes (versus não uso) entre hospitais com 101 a 150 leitos foram, em média, 8,93 vezes as chances observadas em outros hospitais, controlando as demais variáveis.

Também elevadas foram as estimativas do odds ratio do uso de diretrizes nos hospitais com PAC de transplante $(O R=73,52)$, comparados àqueles sem tal PAC, enos hospitais que responderam que realizavam gestão decaso $(O R=29,77)$, comparados aos que não realizavam.

Por fim, na análise multivariada, a presença de UTI mostrou-se com uma associação borderline ( $p=0,0904 ; O R=4,98)$ com a variável dependente contemplada, enquanto a presença de PAC em cirurgia oncológica mostrou-se com uma associação não significante estatisticamente, mas também, certamente, não desprezível ( $p=0,1196$; $\mathrm{OR}=7,47$ ). 
Tabela 3. Distribuição do uso de diretrizes clínicas reportado por prestadores de serviços hospitalares na saúde suplementar ( $n=3799$ ) segundo variáveis selecionadas. Análise bivariada. Brasil, 2006.

\begin{tabular}{|c|c|c|c|c|c|c|c|}
\hline \multirow[b]{3}{*}{ Variável } & \multirow[b]{3}{*}{$\mathrm{n}$} & \multirow[b]{3}{*}{$\%$} & \multicolumn{4}{|c|}{ Uso de diretrizes } & \multirow{3}{*}{ Teste- $\boldsymbol{\chi}^{2}(p)$} \\
\hline & & & \multicolumn{2}{|c|}{$\underset{(n=1961)}{\operatorname{Sim}}$} & \multicolumn{2}{|c|}{$\begin{array}{c}N \text { ão } \\
(n=1838)\end{array}$} & \\
\hline & & & $\mathrm{n}$ & $\%$ & $\mathrm{n}$ & $\%$ & \\
\hline \multicolumn{7}{|l|}{ Macrorregião } & 0,0001 \\
\hline Norte & 189 & 5,0 & 178 & 94,4 & 11 & 5,6 & \\
\hline Nordeste & 720 & 18,9 & 284 & 39,4 & 436 & 60,6 & \\
\hline Sudeste & 1579 & 41,6 & 989 & 62,7 & 590 & 37,3 & \\
\hline Sul & 820 & 21,6 & 412 & 50,2 & 408 & 49,8 & \\
\hline Centro-Oeste & 491 & 12,9 & 98 & 19,9 & 393 & 80,1 & \\
\hline \multicolumn{7}{|c|}{ Hospital próprio de operadora de planos de saúde } & 0,0400 \\
\hline Sim & 266 & 7,0 & 264 & 99,3 & 2 & 0,7 & \\
\hline Não & 3533 & 93,0 & 1697 & 48,1 & 1836 & 51,9 & \\
\hline \multicolumn{7}{|l|}{ Porte do hospital (leitos) } & 0,1298 \\
\hline$\leq 10$ & 217 & 5,7 & 56 & 25,8 & 161 & 74,2 & \\
\hline $11-50$ & 1404 & 37,0 & 563 & 40,1 & 841 & 59,9 & \\
\hline $51-100$ & 1071 & 28,2 & 651 & 60,8 & 420 & 39,2 & \\
\hline $101-150$ & 596 & 15,7 & 444 & 74,5 & 152 & 25,5 & \\
\hline $151-350$ & 499 & 13,1 & 237 & 47,6 & 262 & 52,4 & \\
\hline$>350$ & 12 & 0,3 & 10 & 83,3 & 2 & 16,7 & \\
\hline \multicolumn{7}{|l|}{$\begin{array}{l}\text { Número de operadoras para as quais presta } \\
\text { serviços }\end{array}$} & 0,0324 \\
\hline $1-5$ & 1195 & 31,8 & 325 & 27,2 & 870 & 72,8 & \\
\hline $6-50$ & 2413 & 64,1 & 1446 & 59,9 & 967 & 40,1 & \\
\hline$>50$ & 154 & 4,1 & 153 & 99,4 & 1 & 0,6 & \\
\hline \multicolumn{7}{|l|}{ Classificação da complexidade assistencial } & 0,0009 \\
\hline Geral II & 867 & 22,8 & 716 & 82,6 & 150 & 17,4 & \\
\hline Geral I com UTI & 681 & 17,9 & 535 & 78,6 & 146 & 21,4 & \\
\hline Geral I sem UTI & 1594 & 42,0 & 477 & 29,9 & 1117 & 70,1 & \\
\hline Especializado com UTI & 122 & 3,2 & 122 & 100,0 & 0 & 0,0 & \\
\hline Especializado sem UTI & 535 & 14,1 & 111 & 20,8 & 424 & 79,2 & \\
\hline \multicolumn{7}{|l|}{ Atendimento ao SUS } & 0,0521 \\
\hline Sim & 2733 & 72,0 & 1163 & 42,6 & 1570 & 57,4 & \\
\hline Não & 1066 & 28,0 & 798 & 74,9 & 268 & 25,1 & \\
\hline \multicolumn{7}{|l|}{ Acreditação* } & 0,0158 \\
\hline Não & 3170 & 86,9 & 1409 & 44,5 & 1761 & 55,5 & \\
\hline Em processo & 252 & 6,9 & 252 & 100,0 & 0 & 0,0 & \\
\hline Concluída & 224 & 6,2 & 224 & 100,0 & 0 & 0,0 & \\
\hline \multicolumn{7}{|l|}{ Serviço/núcleo de Epidemiologia } & 0,4793 \\
\hline Sim & 928 & 24,4 & 550 & 59,2 & 378 & 40,8 & \\
\hline Não & 2871 & 75,6 & 1411 & 49,2 & 1460 & 50,8 & \\
\hline \multicolumn{7}{|l|}{ Serviço/núcleo de Qualidade } & 0,0200 \\
\hline Sim & 1484 & 39,1 & 1019 & 68,7 & 465 & 31,3 & \\
\hline Não & 2315 & 60,9 & 942 & 40,7 & 1373 & 59,3 & \\
\hline
\end{tabular}

* Para essas variáveis, não se obteve resposta de todas as operadoras estudadas. 
Tabela 3 (cont.). Distribuição do uso de diretrizes clínicas reportado por prestadores de serviços hospitalares na saúde suplementar ( $n=3799)$ segundo variáveis selecionadas. Análise bivariada. Brasil, 2006.

\begin{tabular}{|c|c|c|c|c|c|c|c|}
\hline \multirow[b]{3}{*}{ Variável } & \multirow[b]{3}{*}{$\mathrm{n}$} & \multirow[b]{3}{*}{$\%$} & \multicolumn{4}{|c|}{ Uso de diretrizes } & \multirow{3}{*}{ Teste- $\boldsymbol{\chi}^{2}(p)$} \\
\hline & & & \multicolumn{2}{|c|}{$\underset{(n=1961)}{\operatorname{Sim}}$} & \multicolumn{2}{|c|}{$\begin{array}{c}\text { Não } \\
(n=1838)\end{array}$} & \\
\hline & & & $\mathrm{n}$ & $\%$ & $\mathrm{n}$ & $\%$ & \\
\hline Prêmio de qualidade* & & & & & & & 0,1497 \\
\hline Sim & 1601 & 42,2 & 1012 & 63,2 & 589 & 36,8 & \\
\hline Não & 2197 & 57,8 & 949 & 43,2 & 1248 & 56,8 & \\
\hline Capacitação de profissionais da área assistencial & & & & & & & 0,1400 \\
\hline Sim & 1255 & 33,0 & 850 & 67,7 & 405 & 32,3 & \\
\hline Não & 2544 & 67,0 & 1111 & 43,7 & 1433 & 56,3 & \\
\hline Gestão de patologia & & & & & & & 0,0077 \\
\hline Sim & 965 & 25,4 & 776 & 80,4 & 189 & 19,6 & \\
\hline Não & 2834 & 74,6 & 1186 & 41,8 & 1649 & 58,2 & \\
\hline Gestão de caso & & & & & & & 0,0000 \\
\hline Sim & 1158 & 30,5 & 1047 & 90,4 & 111 & 9,6 & \\
\hline Não & 2641 & 69,5 & 914 & 34,6 & 1727 & 65,4 & \\
\hline Segunda opinião para procedimentos específicos & & & & & & & 0,7880 \\
\hline Sim & 1485 & 39,1 & 735 & 49,5 & 750 & 50,5 & \\
\hline Não & 2314 & 60,9 & 1226 & 53,0 & 1088 & 47,0 & \\
\hline Estudos estatísticos da variação na prática médica & & & & & & & 0,5925 \\
\hline Sim & 842 & 22,2 & 370 & 44,0 & 472 & 56,0 & \\
\hline Não & 2957 & 77,8 & 1591 & 53,8 & 1366 & 46,2 & \\
\hline Ouvidoria & & & & & & & 0,0109 \\
\hline Sim & 1420 & 37,4 & 1063 & 74,8 & 357 & 25,2 & \\
\hline Não & 2379 & 62,6 & 898 & 37,8 & 1480 & 62,2 & \\
\hline Campanhas de promoção, prevenção e educação & & & & & & & 0,8517 \\
\hline Sim & 1826 & 48,1 & 916 & 50,2 & 910 & 49,8 & \\
\hline Não & 1973 & 51,9 & 1045 & 53,0 & 928 & 47,0 & \\
\hline PAC - cardiovascular & & & & & & & 0,0742 \\
\hline Sim & 748 & 19,7 & 576 & 77,0 & 172 & 23,0 & \\
\hline Não & 3051 & 80,3 & 1385 & 45,4 & 1666 & 54,6 & \\
\hline PAC - cirurgia oncológica & & & & & & & 0,0001 \\
\hline Sim & 1050 & 27,6 & 974 & 92,7 & 76 & 7,3 & \\
\hline Não & 2749 & 72,4 & 987 & 35,9 & 1762 & 64,1 & \\
\hline PAC - neurocirurgia & & & & & & & 0,0009 \\
\hline Sim & 1118 & 29,4 & 968 & 86,6 & 150 & 13,4 & \\
\hline Não & 2681 & 70,6 & 993 & 37,1 & 1688 & 62,9 & \\
\hline PAC - ortopedia & & & & & & & 0,0344 \\
\hline Sim & 2033 & 53,5 & 1350 & 66,4 & 683 & 33,6 & \\
\hline Não & 1766 & 46,5 & 611 & 34,6 & 1155 & 65,4 & \\
\hline PAC - transplantes & & & & & & & 0,0279 \\
\hline Sim & 321 & 8,4 & 319 & 99,4 & 2 & 0,6 & \\
\hline Não & 3478 & 91,6 & 1642 & 47,2 & 1836 & 52,8 & \\
\hline PAC - neonatologia & & & & & & & 0,0104 \\
\hline Sim & 702 & 18,5 & 626 & 89,2 & 76 & 10,8 & \\
\hline Não & 3097 & 81,5 & 1335 & 43,1 & 1762 & 56,9 & \\
\hline
\end{tabular}

* Para essas variáveis, não se obteve resposta de todas as operadoras estudadas. 
Tabela 4. Modelo de regressão logística explicativo do uso de diretrizes clínicos reportado por prestadores de serviços hospitalares na Saúde Suplementar (n=3799). Brasil, 2006.

\begin{tabular}{lrrrrr}
\hline \multicolumn{1}{c}{ Variável } & $\beta$ & Erro-padrão & $p$ & Odds ratio & \multicolumn{1}{c}{ IC95\% } \\
\hline Intercepto & $-2,32$ & 0,89 & 0,0112 & & \\
Porte 101-150 leitos (ref: menos ou mais leitos) & 2,19 & 1,08 & 0,0479 & 8,93 & 1,$02 ; 78,11$ \\
Com UTI (ref: sem) & 1,61 & 0,93 & 0,0904 & 4,98 & 0,$77 ; 32,20$ \\
Com PAC transplante (ref: sem) & 4,30 & 1,37 & 0,0027 & 73,52 & 4,$75 ; 1137,71$ \\
Com PAC cirurgia oncológica (ref: sem) & 2,01 & 1,27 & 0,1196 & 7,47 & 0,$58 ; 95,53$ \\
Gestão de caso (ref: não) & 3,39 & 1,13 & 0,0040 & 29,77 & 3,$10 ; 286,11$ \\
\hline
\end{tabular}

\section{Discussão}

As operadoras e hospitais prestadores apresentam, como esperado, a mesma distribuição geográfica, encontrando-se mais concentrados na região Sudeste e, progressivamente, menos presentes nas regiões Sul, N ordeste, Centro-O este e Norte.

Em termos gerais, as duas pesquisas são estatisticamente representativas da realidade nacional, refletindo, entretanto, eventuais problemas do cadastro utilizado como base para a definição da amostra. Ressalta-se ainda a possibilidade de presença de viés de não-resposta, no estudo das operadoras, em função do elevado número de recusas de participação, considerando o pressuposto de que normalmente quem recusa apresenta piores condições. A pesar dos limites, as pesquisas propiciaram a apreciação de que o uso de diretrizes clínicas e de outras práticas da gestão clínica é ainda muito limitado e incipiente entre operadoras de planos de saúde e hospitais atuantes no mercado de saúde suplementar do país.

0 estudo sobre as operadoras indica que somente $32,3 \%$ das operadoras referiram o uso de diretrizesclínicas entre 2004 e2005, sendo menos freqüente sua utilização no segmento filantrópico $(6,5 \%)$ e das seguradoras $(7,7 \%)$. Especificamente, chamam a atenção os resultados referentes às seguradoras, que possivelmente expressam uma postura assumida de desvinculação do seu papel da assistência à saúde.

0 estudo dos hospitais, relativo ao ano de 2006, apresenta resultados um pouco melhores, pois $51,6 \%$ dos hospitais referiram usar diretrizes clínicas frente aos 32,3\% de operadoras que relataram seu uso.

Ainda em relação às operadoras, há importante variação no uso de diretrizes clínicas e de outras práticas de gestão clínica, tanto geográfi$\mathrm{ca}$, quanto em termos dos segmentos do mercado de saúde suplementar.

O uso de diretrizes clínicas foi mais referido nas regiões Sudeste e Centro- 0 este, o que pode estar associado ao fato das grandes operadoras de abrangência nacional estarem concentradas predominantemente naquelas regiões. Porte e abrangência de atuação parecem incentivar as operadoras a uma maior profissionalização.

A aparente contradição entre a declaração de uso de diretrizes clínicas entreoperadores eprestadores hospitalares no Centro-Oeste pode, na realidade, refletir a concentração de algumas sedes de empresas de autogestão em Brasília. As operadoras de autogestão são as que mais referem o uso de diretrizes. Em contraponto, na região Sul, o relativamente elevado índice de declarações de uso por parte dos prestadores pode ser explicado por um nível de qualificação assistencial dos hospitais, de fato, diferenciado em relação a outras regiões, enquanto que as operadoras lá localizadas são predominantemente locais ou regionais e de menor porte.

Estes resultados de maneira geral evidenciam que 0 uso de diretrizes nos hospitais parece ser mais decorrente da iniciativa de seus dirigentes e grupos médicos do que uma resposta à indução das operadoras para as quais eles prestam serviços.

Vale destacar, entretanto que tanto em relação às operadoras quanto aos prestadores hospitalares, trata-se deum resultado geral, que não discrimina a abrangência de condições e patologias objeto das diretrizes, nem sua origem/fonte - se baseadas em evidências científicas internacionalmente validadas ou se baseadas em consensos de especialistas ou ainda se definidas de outras formas -, nem os mecanismos utilizados de 
difusão, deincentivo e de monitoramento do uso junto aos profissionais e pacientes, etc., o que, dentre outros aspectos, indicaria o grau de profissionalização do processo de uso de diretrizes clínicas nas operadoras e nos hospitais. Considerando estes aspectos, a declaração do uso de diretrizes clínicas deveser relativizada.

No caso das operadoras, chama a atenção o uso limitado de outras ferramentas da gestão da clínica e, nos poucos casos referidos de uso, a não associação com a utilização das diretrizes clínicas, o que seria esperado, já queesta seconstitui no padrão de referência para a aplicação das demais ferramentas. As associações observadas restringem-se a fatores não vinculados à assistência, tais como localização geográfica, tipo e porte da operadora.

Entretanto os resultados encontrados no estudo dos hospitais, além de mais favoráveis, parecem ser mais consistentes, considerando que os prestadores, mais do que as operadoras, apresentaram associações entre o uso de diretrizes clínicas e diversos outros fatores investigados especialmente no âmbito assistencial, como a utilização de outras ferramentas da gestão da clínica, a presença de características estruturais de garantia de qualidade, além do porte e complexidade assistencial do hospital.

Outra importante associação foi com o número de operadoras para as quais os hospitais prestam serviços; quase a totalidade de hospitais que prestam serviços a mais de cinqüenta operadoras declarou o uso de diretrizes clínicas.

M esmo com resultados mais favoráveis para os hospitais, a análise do emprego de diversas práticas eestruturas de gestão assistencial evidencia o quanto ainda é incipiente e insatisfatória a ingerência dos dirigentes dessas organizações operadoras e hospitais - sobre as equipes profissionaise, por conseqüência, sobrea qualidadedos cuidados à saúde providos aos pacientes.

Indiscutivelmente, o desenho dos mecanismos regulatórios no país tem avançado no sentido de valorizar a qualidade da atenção prestada sem perder de vista a eficiência do sistema e dos serviços de saúde públicos e privados. Entretanto, há ainda um longo caminho a ser trilhado para se avançar no processo de regulação da assistência à saúde.

As iniciativas observadas, em operadoras ou em hospitais, no campo da gestão da clínica ainda são recentes no país, além de independentese pulverizadas, não alcançando a necessária articulação entre si para consolidar-se como uma das dimensões da gestão de organizações de saú- de, que associada a outras práticas e ferramentas, deveria ser incorporada como políticas do sistema de saúde, com amplo compromisso com a qualidade dos cuidados de saúde oferecidos.

Iniciativas voltadas para a melhoria da qualidade assistencial precisam ser integradas e conduzidas no nível organizacional ${ }^{18}$. A incorporação de diretrizes clínicas eda gestão da clínicanos serviços de saúde pode ser potencializada, seadequadamente articulada a outras ferramentas gerenciais que, tendo as diretrizes como referência, sejam capazes de impactar sobre a prática médica, com vistas à melhoria da qualidade da atenção. Entre elas, enumeram-se a programação de atividades, o estabelecimento de indicadores e metas assistenciais, a realização de auditorias e de educação continuada junto às equipes e uma estrutura de incentivos que induza as equipes a incorporarem novas práticas e ferramentas que possi bilitem o monitoramento ea melhoria contínua da qualidade assistencial ${ }^{19,20}$.

Por tudo isso, é necessário promover uma mudança cultural para o desenvolvimento da capacidade organizacional no sentido da provisão de cuidados de saúde com foco nos pacientes, na garantia de qualidade e na prestação de contas.

\section{Colaboradores}

M C Portela eSM L Lima trabalharam na concepção teórica e desenvolvimento dos dois estudos contemplados ena redação inicial efinal do artigo. MTL Vasconcellos foi responsável pelo desenho das amostras dos dois estudos. CC Escosteguy, VM B Ferreira e C Brito trabalharam na concepção teórica e desenvolvimento do primeiro estudo considerado. M M Vasconcellos participou da concepção teórica e desenvolvimento do segundo estudo. Todos os autores revisaram 0 artigo. 
Agradecimentos

A pesquisa sobreas operadoras de planos de saúde foi realizada com financiamento da Organização Pan-Americana de Saúde (OPAS) e colaboração da Agência Nacional de Saúde Suplementar (ANS), estando regida pela carta acordo BR/CNT/030.1767, firmada entre a Fundação para o Desenvolvimento Científico eTecnológico em Saúde (FIOTEC) e a OPAS.

A pesquisa sobre os prestadores hospitalares foi realizada com financiamento da Agência $\mathrm{Na}$ cional de Saúde Suplementar (ANS) e os recursos foram administrados pela Fundação para 0 Desenvolvimento Científico e Tecnológico em Saúde (FIOTEC).

Agradecemos ainda a M aria Alicia Dominguez U gá e Silvia Gerschman, pesquisadoras do Departamento de Administração e Planejamento em Saúde da Escola Nacional de Saúde Pública, integrantes da equipe central do Estudo dos prestadores hospitalares frente às práticas de micro-regulação das operadoras de planos de saúde (ANS, 2007), cujos dados são analisados neste artigo. Em especial, a Maria Alicia Dominguez Ugá, que foi coordenadora da referida pesquisa.

Aos demais profissionais quetrabalharam nas duas pesquisas. À equipe de pesquisadores de campo: Águida Wichrowski Kopf; Alda Maria Lacerda da Costa; Carla Lourenço Tavares de Andrade; Cátia Cristina M artins; Flávia H elena M iranda de Araújo Freire; I vone Rodrigues M orallis; Juliana Garcia Gonçalves; M ônica de Oliveira M arques; Rejane Christine de Sousa Queiroz; Simone Cristina da Costa Ferreira; Valdir Zettel; Vanessa A parecida Thomaz; e Vanessa Costa e Silva. Ao responsável pelos bancos de dados: Raulino Sabino da Silva. À secretária executiva: Ana Paula Lucas Caetano.

\section{Referências}

1. Sackett DL, Straus SE, Richardson WS, Rosenberg W, Haynes RB. Evidence-Based M edicine: H ow to Practice and Teach EBM. $2^{\text {nd }}$ ed. London: Churchill Livingstone; 2000.

2. Institute of Medicine. Committee to Advise the Public Health Service on Clinical Practice Guidelines. In: Field MJ, Lohr KN, editors. Clinical Practice Guidelines: Directions for a New Program. Washington, D.C.: National Academy Press; 1990.

3. Bodenheimer T, Wagner EH, Grumbach K. Improving primary care for patients with chronic illness. JAMA 2002; 288:1775-1779.

4. Grimshaw JM, Russell IT. Effect of clinical guidelines on medical practice: a systematic review of rigorous evaluations. Lancet 1993; 342:1317-1322.

5. Grol R, Dalhuijsen J, Thomas S, Veld C, Rutten G, Mokkink $\mathrm{H}$. Attributes of clinical guidelines that influence use of guidelines in general practice: observational study. BMJ 1998; 317: 858-861.

6. Moody-Williams JD, Krug S, O'Connor R, Shook JE, Athey J, Holleran RS. Practice guidelines and performance measures in emergency medical services for children. Ann Emerg M ed 2002; 39:404-412.

7. Shekelle PG, Eccles MP, Grimshaw JM, Woolf SH. When should clinical guidelines be updated? BM J 2001; 23:155-157.

8. Bero LA, Grilli R, Grimshaw JM, Harvey E, Oxman $A D$, Thompson MA. Closing the gap between research and practice: an overview of systematic reviews of interventions to promote the implementation of research findings. BMJ 1988; 317:465-468.

9. Masi CM, Blackman DJ, Peek ME. Interventions to enhance breast cancer screening, diagnosis, and treatment among racial and ethnic minority women. Med Care Res Rev 2007; 64:195S-242S.

10. Merrick EL, Garnick DW, Horgan CM, Hodgkin D. Quality measurement and accountability for substance abuse and mental health services in $M$ anaged Care Organizations. M ed Care 2002; 40:1238-1248.

11. Bennett CL, Somerfield MR, Pfister DG, Tomori C, Yakren S, Bach PB. Perspectives on the value of American Society of Clinical Oncology clinical guidelines as reported by $O$ ncologists and $\mathrm{H}$ ealth $\mathrm{M}$ aintenance Organizations. J Clin Oncol 2003; 21:937-941. 
12. Rask K, Culler S, Scott T, Kohler S, Hawley J, Friedman E, Naylor DV. Adopting National Quality Forum medication safe practices: Progress and barriers to hospital implementation. J Hosp M ed 2007; 2:212-218.

13. Portela MC, Escosteguy CC, Lima SM L, Ferreira, VMB, Vasconcellos MTL, Brito C. Diretrizes Clínicas e outras práticas voltadas para a melhoria da qualidade assistencial em operadoras de planos de saúde sob a perspectiva de seus dirigentes no Brasil. Cad Saúde Pública 2008; 24:253-266.

14. Ugá MAD, Lima SML, Portela MC, Vasconcellos M M, Gerschman S. Estudo dos prestadores hospitalares frente às práticas de micro-regulação das operadoras de planos de saúde. Rio de Janeiro: Escola Nacional de Saúde Pública, Fundação Oswaldo Cruz; 2007. [Relatório de pesquisa apresentado à Agência Nacional de Saúde Suplementar]

15. Hedlin D. A procedure for stratification by an extended Ekman rule. Journal of Official Statistics 2000; 16:15-29.

16. Hájek J. Limiting distribution in simple random sampling from a finite population. Publications of the $M$ athematics Institute of the Hungarian Academy of Science 1960; 5:361-374.

17. Haldane JBS. On a method of estimating frequencies. Biometrika 1945; 33:222-225.

18. Browman GP. Clinical practice guidelines and health care decisions: credibility gaps and unfulfilled promises. Nat Clin Pract Oncol 2005; 2:480-481.

19. Caminiti C, Scoditti U, Diodati F, Passalacqua R. How to promote, improve and test adherence to scientific evidence in clinical practice. BMC Health Serv Res 2005; 5:62.

20. Jamtvedt G, Young JM, Kristoffersen DT, O'Brien $M A$, Oxman AD. Audit and feedback: effects on professional practice and health care outcomes. Cochrane Database Syst Rev 2006; 2:CD 000259.

Artigo apresentado em 11/07/2007

Aprovado em 09/10//2007

Versão final apresentada em 29/02/2008 